\title{
Hidden orbital polarization in diamond, silicon, germanium, gallium arsenide and layered materials
}

\author{
Ji Hoon Ryoo and Cheol-Hwan Park
}

It was previously believed that the Bloch electronic states of non-magnetic materials with inversion symmetry cannot have finite spin polarizations. However, since the seminal work by Zhang et al. (Nat. Phys. 10, 387-393 (2014)) on local spin polarizations of Bloch states in non-magnetic, centrosymmetric materials, the scope of spintronics has been significantly broadened. Here, we show, using a framework that is universally applicable independent of whether hidden spin polarizations are small (e.g., diamond, $\mathrm{Si}, \mathrm{Ge}$ and GaAs) or large (e.g., $\mathrm{MoS}_{2}$ and $\mathrm{WSe}_{2}$ ), that the corresponding quantity arising from orbitalinstead of spin-degrees of freedom, the hidden orbital polarization is (i) much more abundant in nature since it exists even without spin-orbit coupling and (ii) more fundamental since the interband matrix elements of the site-dependent orbital angular momentum operator determine the hidden spin polarization. We predict that the hidden spin polarization of transition metal dichalcogenides is reduced significantly upon compression. We suggest experimental signatures of hidden orbital polarization from photoemission spectroscopies and demonstrate that the current-induced hidden orbital polarization may play a far more important role than its spin counterpart in antiferromagnetic information technology by calculating the current-driven antiferromagnetism in compressed silicon.

NPG Asia Materials (2017) 9, e382; doi:10.1038/am.2017.67; published online 26 May 2017

\section{INTRODUCTION}

Electronic states at a given Bloch wavevector in non-magnetic materials with inversion symmetry are degenerate. Until recently, it was believed that there is no spatial spin distribution if averaged over these two spin-degenerate states. However, it has been found that even in centrosymmetric, non-magnetic crystals, the degenerate Bloch states can have local spin polarization if atoms are not at an inversion center. ${ }^{1}$ Zhang et al. ${ }^{1}$ reported that the lack of the local inversion symmetry at atomic sites leads to hidden, or site-dependent, spin polarization, expanding the scope of spintronics significantly, even to bulk materials with global inversion symmetry.

On the other hand, the orbital contribution to the magnetic moment of solids can be sizable (see e.g., refs 2,3) and even larger than the spin contribution. ${ }^{4}$ The orbital magnetization becomes more important than the spin magnetization in some physical phenomena, for example, current-induced magnetization ${ }^{5}$ and the gyrotropic magnetic effect, ${ }^{6}$ if the spin-orbit coupling (SOC) is weak. Additionally, the important role of orbital polarization in Rashba-split bands ${ }^{7-9}$ and quantum anomalous Hall phases ${ }^{10}$ of systems without inversion symmetry has been explored.

In this paper, we report the finding that the hidden, or sublatticedependent, orbital polarization of Bloch states of centrosymmetric materials can be large (on the order of $\hbar$ ) even without SOC by using the simplest, best-known materials, such as diamond, $\mathrm{Si}$ and $\mathrm{Ge}$, as examples. We describe that, in any non-magnetic, centrosymmetric material, including the aforementioned zinc-blende materials and layered materials such as $\mathrm{MoS}_{2}$ and $\mathrm{WSe}_{2}$, in which the hidden spin polarization is quite large, the hidden spin polarization is completely determined by the interband matrix elements of the site-dependent orbital angular momentum operator. This finding, together with the fact that in materials with weak SOC the hidden spin polarization is small or absent, suggests that the hidden orbital polarization is a more fundamental quantity. We show that the sublattice-dependent spin-orbital texture of centrosymmetric crystals is qualitatively different from that of non-centrosymmetric crystals and that the hidden orbital polarization can play an important role in current-induced magnetization ${ }^{5}$ of both centrosymmetric materials and non-centrosymmetric materials such as GaAs. We then discuss the experimental evidence from photoemission spectroscopies and the technological implications of our findings in antiferromagnetic information technology using current-induced hidden orbital polarizations, which, according to our calculations, could be much more important than their spin counterpart.

\section{MATERIALS AND METHODS}

We calculated the electronic structures of diamond, $\mathrm{Si}$, Ge and GaAs by using a tight-binding model including atomic $s$ and $p$ orbitals $\left(s p^{3} s^{\star} \text { model }\right)^{11}$ and the on-site SOC term $\Delta H_{\mathrm{SOC}}=\left(\alpha^{A} \mathbf{L}^{A} \cdot \mathbf{S}+\alpha^{\bar{A}} \mathbf{L}^{\bar{A}} \cdot \mathbf{S}\right) / \hbar^{2}$, in which $A$ and $\bar{A}$ denote the two sublattices in the zinc-blende structure (see Figure 1b), and $\alpha^{A}$ and $\alpha^{\bar{A}}$ are atomic SOC strengths, and the local orbital angular momentum operator $\mathbf{L}^{\beta}(\beta=A, \bar{A})$ for each sublattice is defined as $L_{i}^{\beta}=-i \hbar \sum_{j, k} \varepsilon_{i j k}\left|p_{j}, \beta\right\rangle\left\langle p_{k}, \beta\right|$, where $\varepsilon_{i j k}$ is the Levi-Civita symbol and $\left|p_{j}, \beta\right\rangle$ is the Bloch sum of $p_{j}$ orbitals at 

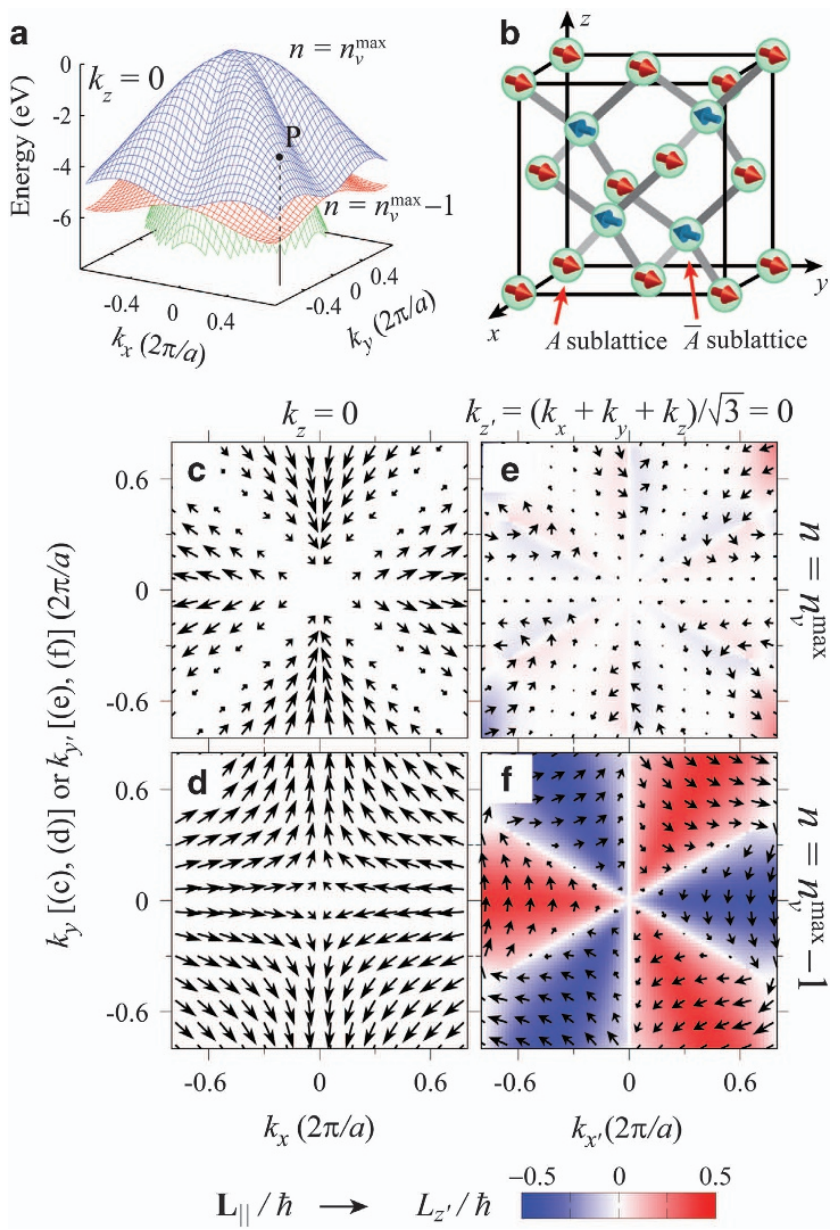

Figure 1 The hidden orbital polarization in diamond without spin-orbit coupling. (a) The electronic band structure of diamond. $n_{v}^{\max }$ is the band index of the highest-energy valence band. (b) The local orbital polarization of state $P$ shown in (a). (c-f) The local orbital texture at the $A$ sublattice of diamond on the $k_{z}=0$ plane $(\mathbf{c}, \mathbf{d})$ and on the $k_{x}+k_{y}+k_{z}=0$ plane $(\mathbf{e}, \mathbf{f})$. The

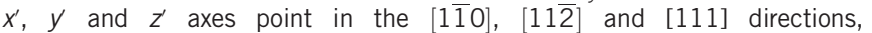
respectively.

sublattice $\beta$. This type of model ${ }^{12}$ has been used in studies of Rashba splitting and spin-orbital textures. ${ }^{7,13,14}$

\section{RESULTS AND DISCUSSION}

First, we discuss the orbital polarization of diamond, whose SOC is negligible. If SOC is neglected, the spin-up and -down states have the same energy and orbital wavefunction. Figure $1 \mathrm{~b}$ shows the local orbital polarization $\left\langle\mathbf{L}^{\beta}\right\rangle_{n \mathbf{k}}=\left\langle n \mathbf{k}\left|\mathbf{L}^{\beta}\right| n \mathbf{k}\right\rangle$ for the orbital part of a Bloch state, $|n \mathbf{k}\rangle$, corresponding to $P$ in Figure 1a. (Note that the expectation values of $\mathbf{L}^{\beta}$ with respect to spin-degenerate states $|n \mathbf{k}\rangle \otimes|\uparrow\rangle$ and $|n \mathbf{k}\rangle \otimes|\downarrow\rangle$ are the same.) Since the product of the inversion operator $(P)$ and the time reversal operator $(T)$ conserves the crystal momentum $\mathbf{k}$, all the Bloch states are invariant under $P T$ operation if we neglect spin. $A$ and $\bar{A}$ are exchanged by $P$. Therefore, the local orbital polarizations at $A$ and $\bar{A}$ are of the same magnitude and are anti-parallel to each other; that is, if we define $\mathbf{L}^{\text {tot }}=\mathbf{L}^{A}+\mathbf{L}^{\bar{A}}$, $\left\langle\mathbf{L}^{\text {tot }}\right\rangle_{n \mathbf{k}}=0$.

Figures 1c-f show $\left\langle\mathbf{L}^{A}\right\rangle_{n \mathbf{k}}$ for the (spin-degenerate) highest-energy valence bands and the second-highest-energy valence bands of diamond, which is on the order of $\hbar$ except on some symmetry lines. Therefore, the orbital polarization on each sublattice of a centrosymmetric material can be large. In the $k_{z}=0$ plane, since $\mathbf{k}$ is invariant under $C_{2} T$, where $C_{2}$ is the operator for $180^{\circ}$ rotation with respect to the $z$-axis, $\left\langle\mathbf{L}^{A}\right\rangle_{n \mathbf{k}}$ lies in the $x y$ plane. We also verified that $\mathrm{Si}$ and $\mathrm{Ge}$ have similar hidden orbital polarization textures (Supplementary Discussion S1).

Interestingly, the hidden orbital polarization can be large even when the total orbital angular momentum is quenched. Note that the total orbital angular momentum is a ground-state property of a crystal, whereas the hidden orbital polarization is a property of quasi-particle excitations and is a function of the Bloch wavevector and the band index. Even in a material where $d$ orbitals of a transition metal element experience a strong octahedral crystal field and the (total) orbital angular momentum is quenched, for example, when the $t_{2 \mathrm{~g}}$ bands are empty/half occupied/fully occupied, a quasi-particle state (either an electron or a hole) from the $t_{2 \mathrm{~g}}$ bands can still have a large hidden orbital polarization.

Next, we show that in non-magnetic, centrosymmetric materials, the hidden spin polarization is a physical quantity completely determined by the site-dependent orbital angular momentum. When SOC is absent, it is apparent that a hidden spin texture cannot exist in these materials; since the electron potential does not depend on the spin, all bands are spin-degenerate, and each Bloch state cannot have a spatially inhomogeneous spin distribution. Conversely, we showed that there can be a large hidden orbital polarization even when SOC is absent. When there is SOC, the spin-up and spin-down bands mix with each other, but they remain degenerate due to $P T$ symmetry. We define the spin or orbital polarization of each band as the average of the expectation values of the two degenerate states. ${ }^{1}$

Let $|n \mathbf{k} s\rangle=|n \mathbf{k}\rangle \otimes|s\rangle$ be spin-degenerate eigenstates of the Hamiltonian without SOC, where $|n \mathbf{k}\rangle$ is the orbital part and $|s\rangle$ is the spin part. In our model, in which SOC is taken into account by $\Delta H_{\mathrm{SOC}}=\alpha\left(\mathbf{L}^{A} \cdot \mathbf{S}+\mathbf{L}^{\bar{A}} \cdot \mathbf{S}\right) / \hbar^{2}$, we can express the local spin polarization $\left\langle\mathbf{S}^{A}\right\rangle_{n \mathbf{k}}^{\text {avg }}=-\left\langle\mathbf{S}^{\bar{A}}\right\rangle^{\text {avg }}$ in terms of the matrix element of the site-dependent orbital angular momentum operator using first-order perturbation theory:

$$
\begin{aligned}
\left\langle\mathbf{S}^{\beta}\right\rangle_{n \mathbf{k}}^{\mathrm{avg}} & =\sum_{\substack{m \neq n \\
s, s^{\prime}}} \frac{\left\langle n \mathbf{k} s\left|P^{\beta \frac{\hbar \sigma}{2}}\right| m \mathbf{k} s^{\prime}\right\rangle\left\langle m \mathbf{k} s^{\prime}\left|\Delta H_{\mathrm{SOC}}\right| n \mathbf{k} s\right\rangle+\text { c.c. }}{2\left(E_{n \mathbf{k}}-E_{m \mathbf{k}}\right)} \\
& =\frac{\alpha}{4} \sum_{m \neq n} \frac{\left\langle n \mathbf{k}\left|P^{\beta}\right| m \mathbf{k}\right\rangle\left\langle m \mathbf{k}\left|\left(\mathbf{L}^{A}+\mathbf{L}^{\bar{A}}\right)\right| n \mathbf{k}\right\rangle+\text { c.c. }}{E_{n \mathbf{k}}-E_{m \mathbf{k}}} \\
& =\frac{\alpha}{2} \sum_{m \neq n} \frac{\left\langle n \mathbf{k}\left|P^{\beta}\right| m \mathbf{k}\right\rangle\left\langle m \mathbf{k}\left|\mathbf{L}^{\beta}\right| n \mathbf{k}\right\rangle+\text { c.c. }}{E_{n \mathbf{k}}-E_{m \mathbf{k}}} .
\end{aligned}
$$

Here, $P^{\beta}$ is the projection operator onto sublattice $\beta, \boldsymbol{\sigma}$ is the Pauli spin matrix, and $E_{n \mathbf{k}}$ is the energy of the state $|n \mathbf{k}\rangle$ when SOC is absent. In the third equality of Equation (1), we have used $\left[\left\langle n \mathbf{k}\left|P^{A}\right| m \mathbf{k}\right\rangle\left\langle m \mathbf{k}\left|\mathbf{L}^{\bar{A}}\right| n \mathbf{k}\right\rangle\right]^{*}=\left\langle n \mathbf{k}\left|P^{A}\right| m \mathbf{k}\right\rangle\left\langle m \mathbf{k}\left|\mathbf{L}^{A}\right| n \mathbf{k}\right\rangle$, which follows from (i) $(P T) P^{A}(P T)^{-1}=P^{\bar{A}}$, $(P T) \mathbf{L}^{A}(P T)^{-1}=-\mathbf{L}^{\bar{A}}, \quad\left(\right.$ iii) $\quad\left\langle n \mathbf{k}\left|P^{A}\right| m \mathbf{k}\right\rangle=-\left\langle n \mathbf{k}\left|P^{\bar{A}}\right| m \mathbf{k}\right\rangle \quad$ if $n \neq m$ and (iv) $P T|n \boldsymbol{k}\rangle$ is equal to $|n \boldsymbol{k}\rangle$ up to a phase factor (recall that $|n \boldsymbol{k}\rangle$ is the orbital part of the wavefunction). We can then calculate the hidden spin polarization from the site-dependent orbital angular momentum operator using Equation (1), one of our key results.

It is straightforward to extend Equation (1) and calculate higherorder terms in a regime where SOC is not small; even in this regime, the interband matrix elements of the orbital angular momentum operator determine the hidden spin polarization. Additionally, 


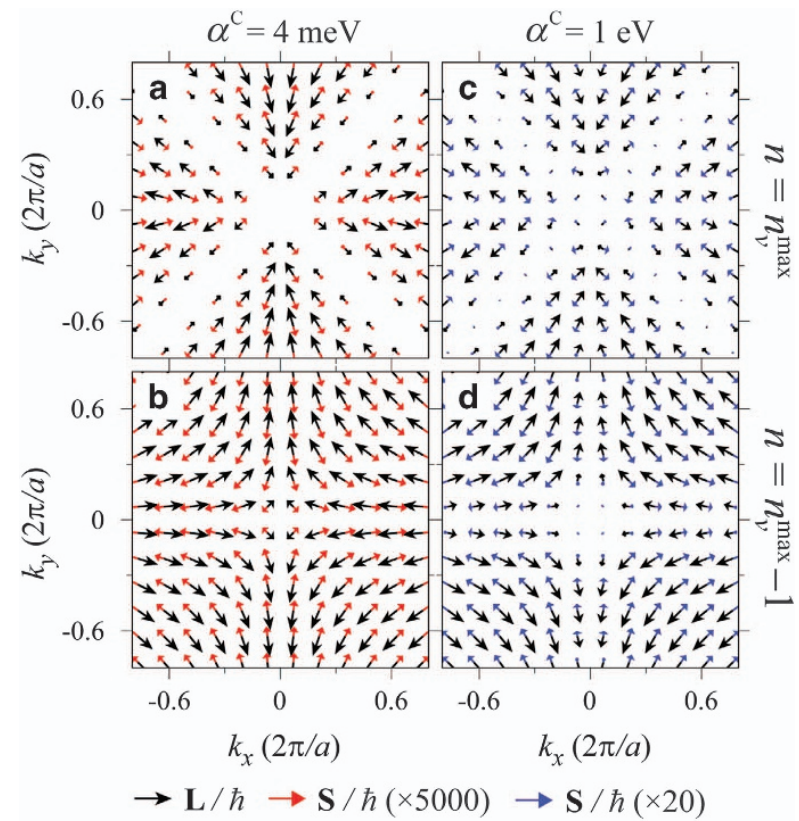

Figure 2 The local spin-orbital texture $\left(k_{z}=0\right)$ at sublattice $A$ of the Bloch states of diamond obtained by using $\alpha^{C}=4 \mathrm{meV}$ (the physical value for diamond) and $\alpha^{C}=1 \mathrm{eV}$.

Equation (1) can be easily extended to materials with more than two atoms per unit cell or to cases involving $d$ or higher- $l$ orbitals. (We can use the known matrix elements of $L_{x}=\left(L_{+}+L_{-}\right) / 2$, $L_{y}=\left(L_{+}-L_{-}\right) / 2 i$ and $L_{z}$ in $|l m\rangle$ basis. These $|l m\rangle$ basis functions can be expressed using cubic harmonics; for example, $|l=2, m= \pm 2\rangle=\left(\left|d_{x^{2}-y^{2}}\right\rangle \pm i\left|d_{x y}\right\rangle\right) / \sqrt{2}, \quad|l=2, m= \pm 1\rangle=$ $\left(\mp\left|d_{x z}\right\rangle-i\left|d_{y z}\right\rangle\right) / \sqrt{2}$ and $|l=2, m=0\rangle=\left|d_{z^{2}}\right\rangle$.)

Figure 2 shows the $\left\langle\mathbf{S}^{A}\right\rangle_{n \mathbf{k}}^{\text {avg }}$ of the two highest-energy valence bands of diamond, calculated by direct diagonalization of the Hamiltonian (rather than using Equation (1)). Since the SOC in diamond is very weak, $\left|\left\langle\mathbf{S}^{A}\right\rangle_{n \mathbf{k}}^{\text {avg }}\right| \ll\left|\left\langle\mathbf{L}^{A}\right\rangle_{n \mathbf{k}}^{\text {avg }}\right|$ (Figures 2a and b). Quite surprisingly, even if we set the SOC strength $\alpha^{C}$ to $1 \mathrm{eV}$, approximately 250 times the physical value, the hidden spin polarization is still an order of magnitude smaller than the orbital polarization (Figures $2 \mathrm{c}$ and $\mathrm{d}$ ) because in diamond, the $A$ and $\bar{A}$ sublattices are strongly coupled to each other. This fact demonstrates that although the conditions for the existence of hidden orbital and spin polarizations are the same in terms of symmetry, it is more difficult for hidden spin polarizations to be appreciably large (see the analysis on $\mathrm{MoS}_{2}$ and $\mathrm{WSe}_{2}$ below and Supplementary Discussion S3). However, in some centrosymmetric materials, the hidden spin polarization can be nearly fully polarized; ${ }^{1}$ even in this case, our claim that the orbital polarization determines the spin polarization is valid. It is noteworthy that the hidden spin polarization shown in Figure 2 is almost identically reproducible by Equation (1), and the lowest-order result in Equation (1) holds for a wide range of SOC strengths up to $\alpha^{C}=1 \mathrm{eV}$.

Interestingly, the directions of spin and orbital polarizations are exactly opposite each other (Figure 2). It is difficult to find a simple reason for this (anti-)alignment because Equation (1) expresses the hidden spin polarization in terms of the off-diagonal matrix elements of $\mathbf{L}^{A}$, rather than the diagonal ones. However, we can understand this behavior in some limited cases (Supplementary Discussion S4).

The hidden spin polarizations in the materials considered above (diamond, silicon and germanium) are much less than $1 \%$, and even if we hypothetically increase the strength of the SOC of carbon atoms to over $1 \mathrm{eV}$ in our tight-binding model calculations (the physical value of SOC is $4 \mathrm{meV}$ ), the hidden spin polarization does not exceed $5 \%$. In contrast, the hidden spin polarizations in $\mathrm{MoS}_{2}$ and $\mathrm{WSe}_{2}$, whose atomic SOC values are only 0.08 and $0.29 \mathrm{eV}$, respectively, are nearly fully polarized. We investigate this phenomenon and find the origin of such large hidden spin polarizations in $\mathrm{MoS}_{2}$ or $\mathrm{WSe}_{2}$ by extending the analysis on diamond, silicon and germanium. Our tight-binding model is based on ref. 15 .

The unit cell of bulk $\mathrm{MoS}_{2}$ consists of two $\mathrm{MoS}_{2}$ units, which are inversion partners (see Figure $3 \mathrm{a}$ ). The spin (or orbital) polarization of the upper layer in the unit cell points in the opposite direction from that of the lower layer. Figure $3 \mathrm{~d}$ shows this hidden spin polarization of the lower layer of $\mathrm{MoS}_{2}$ in the highest-energy valence bands at $K$ as a function of the atomic SOC of Mo atoms. The hidden spin polarization is $80 \%$ polarized at the physical value of $\operatorname{SOC}\left(\alpha_{\text {phys }}^{\text {Mo }}=0.08 \mathrm{eV}\right)$, which is much larger than the hidden spin polarization of diamond $(\sim 0.01 \%)$.

Now, consider the energy splitting among the highest-energy valence states at $K$ due to SOC if there was no interlayer coupling, which we call $2 \alpha(K)^{\mathrm{MoS}_{2}}$, and study how the hidden spin polarization varies with $\alpha(K)^{\mathrm{MoS}_{2}}$ (see Figure 3b). For example, $\alpha(K)^{\mathrm{MoS}_{2}}=0.073 \mathrm{eV}$ if $\alpha^{\mathrm{Mo}}=\alpha_{\text {phys }}^{\mathrm{Mo}}=0.084 \mathrm{eV}$ is used. On the other hand, at zero $\alpha(K)^{\mathrm{MoS}_{2}}$, which is simulated by $\alpha^{\mathrm{Mo}}=0$, there is an energy splitting of $0.11 \mathrm{eV}$ at the top of the valence bands at $K$ among the degenerate doublets due to interlayer splitting (see Figure 3c). Let us call half of this energy splitting $t(K)_{\text {inter }}^{\mathrm{MoS}_{2}}(=0.053 \mathrm{eV})$, which vanishes if we set interlayer hopping integrals to zero. Now if $\alpha(K)^{\mathrm{MoS}_{2}}$, which is proportional to the atomic SOC $\alpha^{\mathrm{Mo}}$, is lower than $t(K)_{\text {inter }}^{\mathrm{MoS}_{2}}$, the hidden spin polarization is roughly proportional to $\alpha^{\mathrm{Mo}}$, which is consistent with Equation (1) (see Figure $3 \mathrm{~d}$ ). At $\alpha(K)^{\mathrm{MoS}_{2}}$ values higher than $t(K)_{\text {inter }}^{\mathrm{MoS}_{2}}$, the wavefunctions of nearby bands are inter-mixed by the SOC, and the hidden spin polarization saturates with $\alpha^{\mathrm{Mo}}$ to $100 \%$ (see Figure 3d). We now can qualitatively understand the results in Figure $3 \mathrm{~d}$.

To deepen our understanding of the hidden orbital and spin polarizations in $\mathrm{MoS}_{2}$, we hypothetically decreased the interlayer distance between each $\mathrm{MoS}_{2}$ layer by $15 \%$ and modified the interlayer hopping integrals according to the scheme in ref. 15 The hidden spin polarization of the highest-energy valence bands (doublet) at $K$ is plotted in Figure 3e as a function of $\alpha^{\mathrm{Mo}}$. In this case, the hidden spin polarization is only $20 \%$ of the fully polarized value and scales linearly with $\alpha^{\mathrm{Mo}}$ around $\alpha^{\mathrm{Mo}}=\alpha_{\text {phys }}^{\mathrm{Mo}}=0.084 \mathrm{eV}$. Note that as a byproduct, our calculations give us insight into $\mathrm{MoS}_{2}$ under a high pressure. A 15\% compression of transition metal dichalcogenide compounds has already been achieved in recent high-pressure experiments; ${ }^{16}$ the hidden spin polarization of $\mathrm{MoS}_{2}$ under pressure is likely to be significantly lower than that of $\mathrm{MoS}_{2}$ not under pressure. To obtain a quantitative prediction, first-principles calculations with structural optimizations are necessary. Although such first-principles calculations are beyond the scope of this study, the qualitative prediction of the reduction of hidden spin polarizations in inversion-symmetric transition metal dichalcogenides due to pressure remains meaningful.

We now perform a similar analysis on $\mathrm{WSe}_{2}$. In this material, $\alpha(K)_{\text {phys }}^{\mathrm{Wse}_{2}}=0.25 \mathrm{eV}$ (Figure $4 \mathrm{a}$ ), and $t(K)_{\text {inter }_{2}}^{\mathrm{Wse}_{2}}=0.036 \mathrm{eV}$ (Figure $4 \mathrm{~b}$ ): the SOC is stronger in $\mathrm{Wse}_{2}$ than in $\mathrm{MoS}_{2}$, and the interlayer coupling is weaker in $\mathrm{WSe}_{2}$ than in $\mathrm{MoS}_{2}$. Both of these differences lead to stronger hidden spin polarizations in $\mathrm{WSe}_{2}$ than in $\mathrm{MoS}_{2}$, in agreement with the results of our calculations (Figures $4 \mathrm{c}$ and $\mathrm{d}$ ).

Although Equation (1), which is the result of a perturbation theory calculation, is not applicable per se when the SOC is stronger than the interlayer coupling of $\mathrm{MoS}_{2}$ and $\mathrm{WSe}_{2}$, it is still true that the site- 

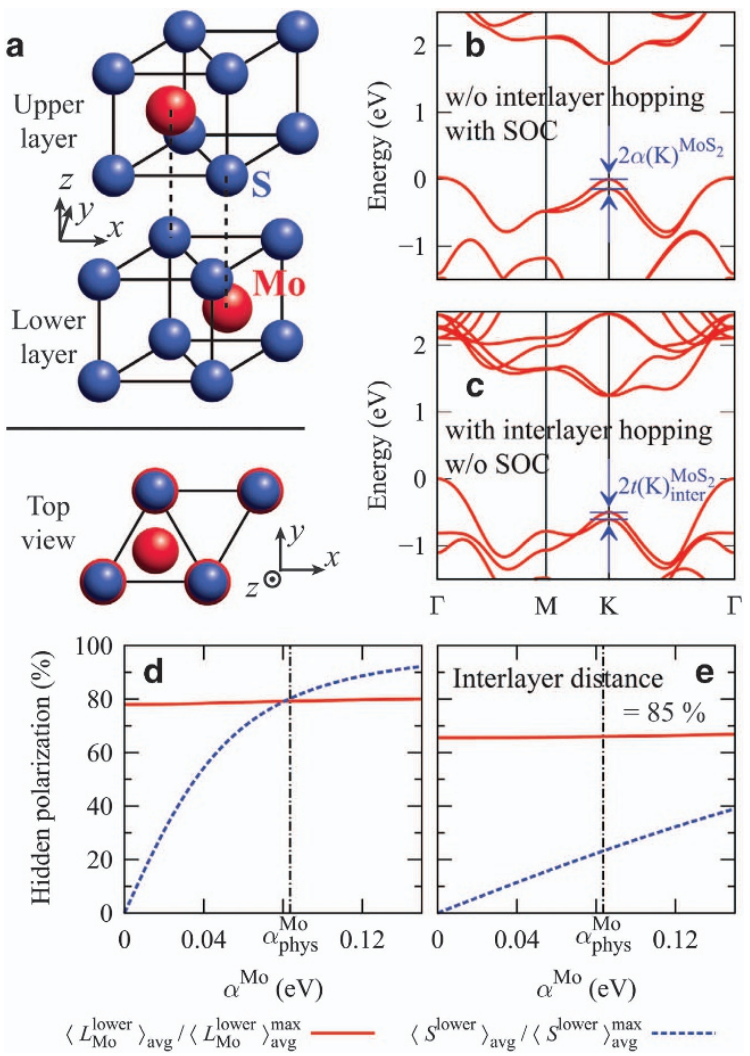

Figure 3 (a) The atomic structure of bulk $\mathrm{MoS}_{2}$. (b,c) The electronic band structures of $\mathrm{MoS}_{2}$ obtained by setting the interlayer coupling (b) or the atomic SOC (c) to zero. (d) The hidden polarizations along $z$ of the lower layer of the highest-energy valence bands at $\mathrm{K}$ versus atomic SOC. The fully polarized values are $\left\langle S^{\text {lower }}\right\rangle_{\text {avg }}^{\max }=\frac{h}{2} \times 0.5$ and $\left\langle L^{\text {lower }}\right\rangle_{\text {avg }}^{\max }=2 \hbar \times 0.5$. The vertical dash-dotted line shows the physical value of the atomic spin-orbit coupling of Mo atoms. (e) The same quantity as in (d), but with the interlayer distance reduced to $85 \%$ of the actual value.

dependent orbital angular momentum determines the hidden spin polarization. In the case of bulk $\mathrm{MoS}_{2}$ and $\mathrm{WSe}_{2}$, because both the strength of the SOC and the interlayer coupling are smaller than the intralayer coupling, we can determine the hidden spin polarization from the hidden orbital polarization by treating both the strength of the SOC and the interlayer coupling as perturbations:

$$
\left\langle S^{\text {lower }}\right\rangle_{\text {avg }}=\left\langle S^{\text {lower }}\right\rangle_{\text {avg }}^{\max } \times \frac{\alpha^{\mathrm{MoS}_{2}}(K)}{\left(t_{\text {inter }}^{\operatorname{MoS}_{2}}(K)^{2}+\alpha^{\operatorname{MoS}_{2}}(K)^{2}\right)^{1 / 2}}
$$

as explained in Supplementary Discussion S2. As mentioned previously, this result can also be obtained by using higher-order perturbation theory only with respect to SOC; in hindsight, expanding the above equation, we know that these higher-order terms should coincide term by term with

$$
\left\langle S^{\text {lower }}\right\rangle_{\text {avg }}=\left\langle S^{\text {lower }}\right\rangle_{\text {avg }}^{\max } \times\left[\frac{\alpha^{\mathrm{MoS}_{2}}(K)}{t_{\text {inter }}^{\mathrm{MoS}_{2}}(K)}-\frac{1}{2}\left(\frac{\alpha^{\mathrm{MoS}_{2}}(K)}{t_{\text {inter }}^{\mathrm{MoS}_{2}}(K)}\right)^{2}+\cdots\right] .
$$

In contrast with $\mathrm{MoS}_{2}$ or $\mathrm{WSe}_{2}$, in which the two subsystems comprising the unit cell are weakly coupled, the two sublattices of diamond or silicon are strongly coupled to each other; hence, the typical energy separation between energy bands is on the order of the nearest-neighbor hopping integral (a few eVs) and is much larger than the SOC. Remarkably, we can now understand, from the same principles, why the hidden spin polarization in diamond, silicon or germanium is very small and why that in $\mathrm{MoS}_{2}$ or $\mathrm{WSe}_{2}$ is very large.

Now, we turn our attention to the hidden orbital polarization. While the hidden spin polarization depends strongly on the strength of the SOC, the hidden orbital polarization is rather insensitive to it (see Figures 3d,e,4c and d). The reason is twofold: (1) the hidden orbital polarization is already large without the SOC and (2) the two highest-energy valence band doublets at $K$ have approximately the same hidden orbital polarization. This supports our primary claim that hidden orbital polarizations are much more widespread in nature than their spin counterparts. The hidden spin polarization is large only if an inversion center is not located at an atomic site and the SOC is stronger than the energy separation between the bands of interest and other nearby bands. In contrast, the hidden orbital polarization can be large in general if only the first (symmetry-related) condition is met. Our analysis of the connection between the hidden spin and orbital polarizations in $\mathrm{MoS}_{2}$ and $\mathrm{WSe}_{2}$ has not been performed in previous studies, in which the focus has been solely on the hidden spin polarization.

Thus far, we have discussed the hidden spin and orbital polarizations of centrosymmetric materials. The spin texture in non-centrosymmetric materials is qualitatively different from that in centrosymmetric systems. Without SOC, all the electronic energy bands of a non-magnetic material are spin degenerate. Contrary to centrosymmetric systems, in which the SOC intermixes spin-up and spin-down components without lifting the degeneracy, the SOC in non-centrosymmetric systems lifts this degeneracy.

Figures $5 \mathrm{a}-\mathrm{d}$ show the spin-orbital texture $\left(k_{z}=0\right)$ of the two bands of GaAs split from the second-highest, spin-degenerate valence bands when SOC is absent. The orbital polarization of As atoms is approximately twice that of $\mathrm{Ga}$ atoms, which can be attributed to the lower on-site potential energy of As atoms. Their directions are opposite to each other, similar to the hidden orbital polarizations at the $A$ and $\bar{A}$ sublattices of diamond (Figures 1 and 2).

Comparing the upper spin-split band (Figures $5 \mathrm{a}$ and $\mathrm{b}$ ) and the lower spin-split band (Figures $5 \mathrm{c}$ and d), we note that, except near the $k_{x}$ or $k_{y}$ axes, the orbital polarizations of the upper and lower bands are approximately the same because the SOC mixes only the spin-up and -down bands together; its magnitude is smaller than the energy distance from those bands to other adjacent bands.

The spin texture of GaAs in Figure 5 demonstrates the following features: (i) excluding the regions near $k_{x}=0$ or $k_{y}=0$ where four bands are degenerate if the SOC is absent, the spin polarization is parallel or anti-parallel to the orbital polarization, (ii) the spin polarization at the $\mathrm{Ga}$ atoms and As atoms of each spin-split band are parallel to each other, in contrast with the hidden spin polarization of diamond, that is, $\left\langle\mathbf{S}^{A}\right\rangle_{n \mathbf{k}}^{\text {avg }}$ and $\left\langle\mathbf{S}^{\bar{A}}\right\rangle_{n \mathbf{k}}^{\text {avg }}$ are anti-parallel to each other, and (iii) the spin is almost fully polarized in each band. These observations also hold for other bands of GaAs (Supplementary Discussion S1).

These features can be explained as follows. When the SOC is neglected, the spin-up and -down bands are degenerate and share the common orbital wavefunction $|n \mathbf{k}\rangle$. Within degenerate perturbation theory, the effect of the SOC is described by diagonalizing $\Delta H_{\mathrm{SOC}}=\left(\alpha^{\mathrm{Ga}} \mathbf{L}^{\mathrm{Ga}}+\alpha^{\mathrm{As}} \mathbf{L}^{\mathrm{As}}\right) \cdot \mathbf{S} / \hbar^{2}$ in the two-dimensional Hilbert space spanned by the spin-up and spin-down states. (We set $\alpha^{\mathrm{Ga}}=0.12 \mathrm{eV}$ and $\alpha^{\mathrm{As}}=0.28 \mathrm{eV} \cdot{ }^{17}$ ) Therefore, if there is no other degeneracy, the direction of the spin polarization of one spin-split band is parallel to $\left\langle n \mathbf{k}\left|\left[\alpha^{\mathrm{Ga}} \mathbf{L}^{\mathrm{Ga}}+\alpha^{\mathrm{As}} \mathbf{L}^{\mathrm{As}}\right]\right| n \mathbf{k}\right\rangle$ (we will denote a unit vector aligned in this direction as $\hat{\rho}_{n k}$ ) and the spin polarization of the other spin-split band points in the opposite direction. We define 

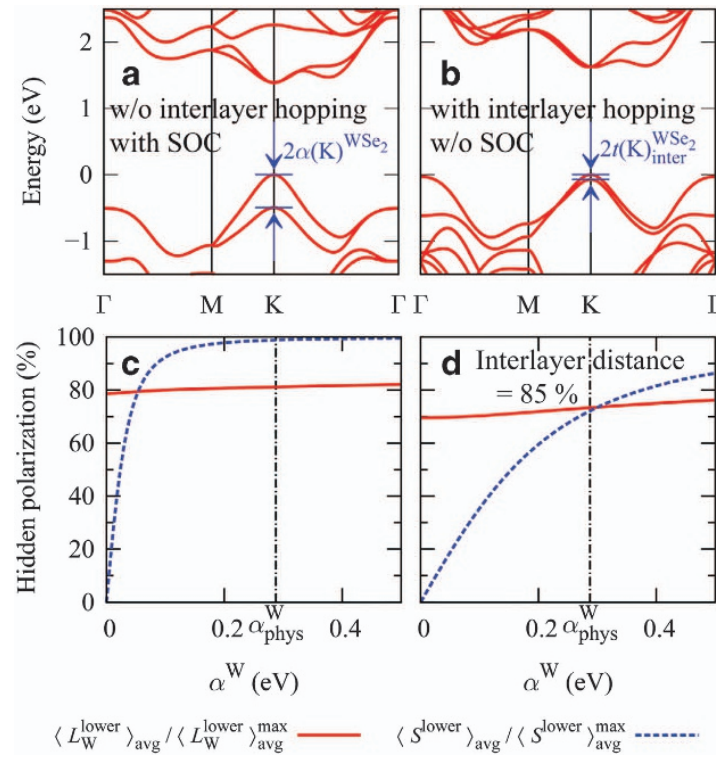

Figure 4 (a, b) The electronic band structures of $\mathrm{WSe}_{2}$ obtained by setting the interlayer coupling to zero (a) or the atomic SOC (b) to zero. (c) The hidden polarizations along $z$ of the lower layer of the highest-energy valence bands at $\mathrm{K}$ versus atomic SOC. The fully polarized values are $\left\langle S^{\text {lower }}\right\rangle_{\text {avg }}^{\max }=\frac{\hbar}{2} \times 0.5$ and $\left\langle L^{\text {lower }}\right\rangle_{\text {avg }}^{\max }=2 \hbar \times 0.5$. The vertical dash-dotted line shows the physical value of the atomic spin-orbit coupling of $\mathrm{W}$ atoms. (d) The same quantity as in (d), but with the interlayer distance reduced to $85 \%$ of the actual value.

$\left|\uparrow ; \hat{\rho}_{n \mathbf{k}}\right\rangle$ and $\left|\downarrow ; \hat{\rho}_{n \mathbf{k}}\right\rangle$ as the spinors whose spin quantization axes are parallel to and anti-parallel to $\hat{\rho}_{n k}$, respectively. Then, the wavefunctions of the spin-split bands are $|n \mathbf{k}\rangle \otimes\left|\uparrow ; \hat{\rho}_{n \mathbf{k}}\right\rangle$ and $|n \mathbf{k}\rangle \otimes\left|\downarrow ; \hat{\rho}_{n \mathbf{k}}\right\rangle$. Therefore, the spin is nearly fully polarized in each spin-split band, and the spin polarizations at $\mathrm{Ga}$ atoms and As atoms are parallel to each other.

We can further understand the direction of the spin polarization of each spin-split band. Since $\left\langle\mathbf{L}^{\mathrm{Ga}}\right\rangle_{n \mathbf{k}}$ is anti-parallel to $\left\langle\mathbf{L}^{\mathrm{As}}\right\rangle_{n \mathbf{k}}$ and both the orbital polarization and the atomic SOC of As are larger than those of $\mathrm{Ga}, \hat{\rho}_{n \mathbf{k}}$ is parallel to $\left\langle\mathbf{L}^{\mathrm{As}}\right\rangle_{n \mathbf{k}}$. Hence, the spin of the electronic states in the upper spin-split band, at both sublattices, aligns with $\left\langle\mathbf{L}^{\mathrm{As}}\right\rangle_{n \mathbf{k}}$, and that in the lower spin-split band anti-aligns with $\left\langle\mathbf{L}^{\mathrm{As}}\right\rangle_{n \mathbf{k}}$ (Figure 5). This behavior is different from the hidden spin polarization in centrosymmetric materials, in which the spin polarizations at the two sublattices are opposite to each other.

In addition, in GaAs or other non-centrosymmetric materials, if we decrease the strength of the SOC, the spin polarization of a spin-split band does not change appreciably because the eigenvectors of the full Hamiltonian are independent of the scaling of the spin-orbit interaction Hamiltonian in the small SOC limit. This behavior is different from the case of the hidden spin polarization in centrosymmetric materials, in which the magnitude scales linearly with the strength of the SOC in the same limit (Equation (1) and Figure 2).

Despite the fact that GaAs lacks inversion symmetry, its transport properties are effectively determined by the average of the spin-split bands depending on the level of impurity and temperature. For this reason, the $j=3 / 2$ Luttinger model $^{12}$ is commonly adopted in studying the transport properties of GaAs (e.g., see ref. 18). Although each spin-split band of GaAs is nearly fully spin polarized (Figures $5 a-d$ ), when we average the spin polarization over the two spin-split bands, the spin polarization is very much reduced, but the orbital polarization is almost invariant upon averaging (Figures $5 \mathrm{e}$ and $\mathrm{f}$ ). The averaged spin and orbital polarizations at As atoms (Figure $5 \mathrm{f}$ ) are similar to the hidden spin and orbital polarizations at sublattice $A$ in diamond (Figure 2b). In all cases, including diamond with a very large SOC of $\alpha^{C}=1 \mathrm{eV}$ (Figure 2d), the band-averaged site-dependent spin polarization is much smaller in magnitude than the band-averaged site-dependent orbital polarization. These results indicate that site-dependent orbital polarizations are important in current-induced magnetization ${ }^{5}$ of both centrosymmetric and non-centrosymmetric materials.

Recently, spin-polarized photocurrents were measured from bulk $\mathrm{WSe}_{2},{ }^{19}$ a non-magnetic, centrosymmetric material. The results confirm the hidden spin polarization and the hidden orbital polarization, as the former is generated from the latter. Moreover, the hidden orbital polarization in materials with a small SOC can also be observed by measuring the spin-integrated photocurrents because it is not the spin polarization but the orbital polarization that determines the coupling between electrons and photons. Provided that the final state is well approximated by $s$-like states, the hidden orbital polarization also manifests itself in the circular dichroism of a non-magnetic, centrosymmetric material.

We now discuss the technological implications of our findings. When an electric current is applied to a centrosymmetric material, non-equilibrium, site-dependent orbital and spin magnetization can be generated. The current-induced magnetization is antiferromagnetic due to the nature of the hidden orbital and spin polarizations, and its direction depends on the direction of the current. (We note that a symmetry lowering, for example by strain, is required for diamond, $\mathrm{Si}, \mathrm{Ge}$ and $\mathrm{GaAs}$ to generate a current-induced, site-dependent magnetization; ${ }^{20}$ on the other hand, there are other classes of materials that do not require an additional symmetry lowering including $\mathrm{CuMnAs}^{21}$ or $\mathrm{Mn}_{2} \mathrm{Au},{ }^{22}$ which are also intrinsically antiferromagnetic.) Antiferromagnetic spintronic devices, in which a current generates sublattice-dependent spin-orbit torques and changes the magnetic state of a material, have several advantages over conventional spintronic devices based on ferromagnetism. Since the total magnetic moment of an antiferromagnet is zero, antiferromagnetic devices are largely insensitive to the external environment and do not introduce magnetic crosstalk. Additionally, they operate much faster than ferromagnetic devices. ${ }^{20}$

The concept of hidden orbital polarization established here should be considered in properly predicting the site-dependent magnetism because, as we have shown, the spin polarization of a Bloch state could be much smaller than the orbital polarization in many materials (e.g., see Figures 2 and 5e,f). Moreover, even in materials with weak SOC, the hidden orbital polarization can be used in antiferromagnetic information storage and processing because of the exchange interactions between localized, hidden orbital moments. (Details on the exchange interactions between orbital moments can be found in, for example, refs 23,24)

To illustrate the idea that the current-induced hidden orbital polarization can play a more important role than the hidden spin polarization, we looked into the current-driven antiferromagnetism of silicon under a $2 \%$ uniaxial compressive strain along the [001] direction, achievable in real experiments. ${ }^{25,26}$ (Because silicon has many point group symmetries, an electric current in silicon does not generate site-dependent magnetization; however, a strain can result in current-induced magnetization by breaking some symmetries. ${ }^{20}$ ) Although silicon may not be the best material for antiferromagnetic information technology applications, it is one of the simplest and most well-known materials, a good candidate for supporting our hypothesis. 


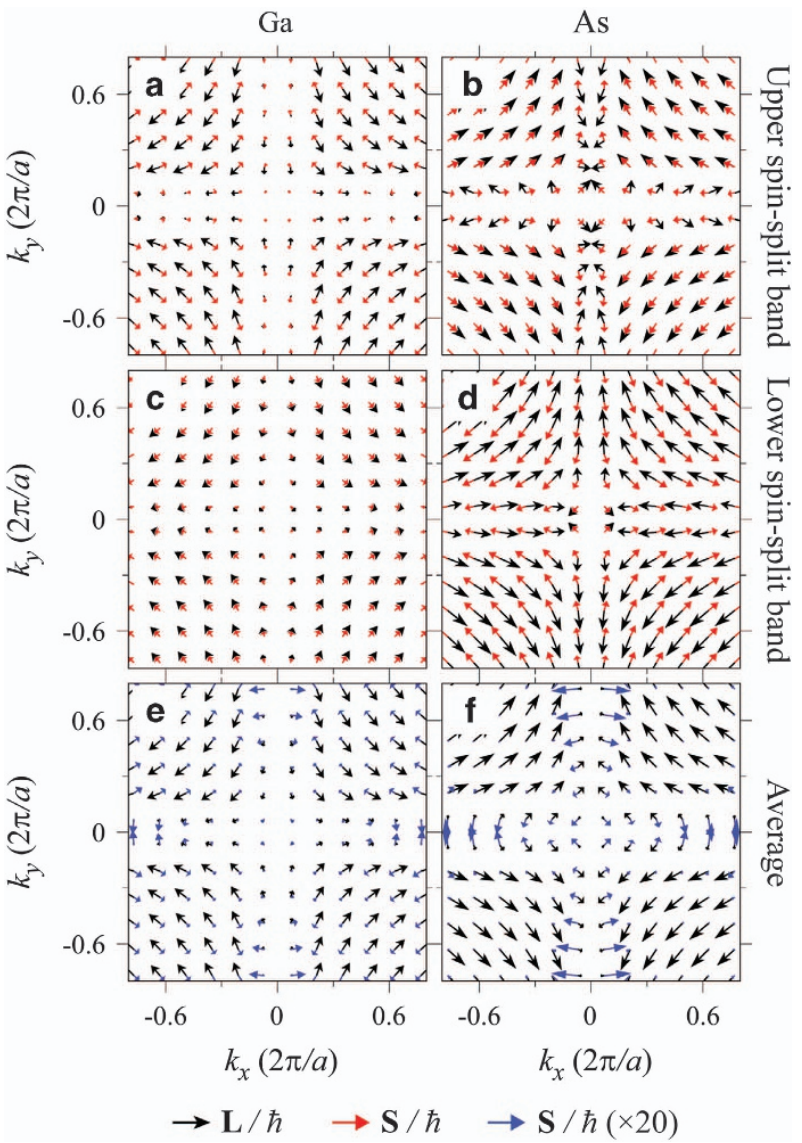

Figure 5 The site-dependent spin and orbital polarizations $\left(k_{z}=0\right)$ of the two bands of GaAs that originate from the spin-degenerate, second-highestenergy doublet among valence bands when SOC is neglected. (a) and (b), (c) and (d), and (e) and (f) show the quantities of the upper spin-split band, those of the lower spin-split band, and their averages, respectively.

The effect of strain is simulated within our tight-binding model using Harrison's universal scaling method. ${ }^{27}$ Following ref. 5, we obtain the non-equilibrium occupation factor $f_{v \mathbf{k}}^{\prime}$ of a Bloch state $|v \mathbf{k}\rangle$ by considering the change from the equilibrium Fermi-Dirac occupation factor $f_{v \mathbf{k}}=f_{\mathrm{FD}}\left(E_{v \mathbf{k}}\right)$ of each Bloch state with the energy eigenvalue $E_{v k}$ :

$$
f_{v \mathbf{k}}^{\prime}=f_{v \mathbf{k}}+\frac{e \boldsymbol{\varepsilon} \tau}{\hbar} \cdot \frac{\mathrm{d} E_{v \mathbf{k}}}{\mathrm{d} \mathbf{k}} \frac{\mathrm{d} f_{\mathrm{FD}}\left(E_{v \mathbf{k}}\right)}{\mathrm{d} E_{v \mathbf{k}}}
$$

where $\tau$ denotes the scattering lifetime of charge carriers, $e$ the absolute value of the charge of an electron and $\varepsilon$ the applied electric field. The current-induced, site-dependent magnetization at the $A$ sublattice, $\mathbf{M}^{A}$, is then given by

$$
\mathbf{M}^{A}=-\frac{\mu_{\mathrm{B}}}{\hbar} \sum_{v} \int_{B Z} \frac{d^{3} k}{(2 \pi)^{3}} f_{v \mathbf{k}}^{\prime}\left(\left\langle\mathbf{L}^{A}\right\rangle_{v \mathbf{k}}+2\left\langle\mathbf{S}^{A}\right\rangle_{v \mathbf{k}}\right)
$$

where $\mu_{\mathrm{B}}$ is the Bohr magneton. As in ref. 5, we assumed that the spin $g$-factor of electrons is 2 .

Figure 6 shows the calculated contributions of the orbital and spin polarizations to the induced magnetization of strained, hole-doped silicon at sublattice $A$ per unit strength of the electric field as a function of the doping concentration $n_{p}$. The scattering lifetime $\tau$ at each $n_{p}$ is extracted from the measured mobility data ${ }^{28}$ by using the Drude model. a

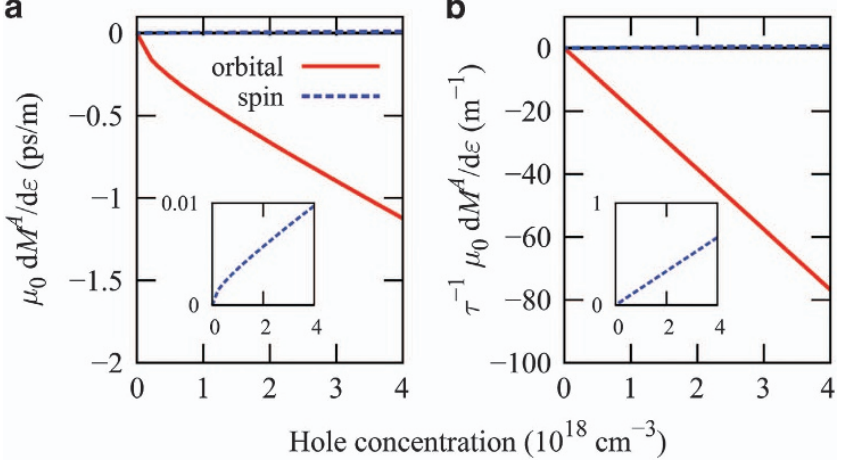

Figure 6 (a) The orbital and spin contributions to the current-induced, site-dependent magnetization of silicon under a $2 \%$ uniaxial compressive strain along [001] versus the hole concentration $n_{p}$. (b) The same quantity divided by the scattering lifetime of holes.

Clearly, the orbital contribution to the current-induced antiferromagnetism is much larger than the spin contribution. Additionally, the induced magnetization at each site of silicon can be larger than the total induced magnetization of $\mathrm{Cr}_{2} \mathrm{O}_{3}$, the most well-known magnetoelectric material, with a $\mu_{0} \mathrm{~d} M / \mathrm{d} \varepsilon$ value of approximately $1 \mathrm{ps} \mathrm{m}^{-1} .^{29}$ Again, we are not claiming that compressed silicon is the best material for antiferromagnetic information technology exploiting the hidden orbital polarization; larger current-induced antiferromagnetism is expected in lower-symmetry materials. However, our proof-ofconcept calculations illustrate that it is a worthwhile research direction to search for materials with large hidden orbital polarizations useful in antiferromagnetic information technology, irrespective of the size of the SOC. This result shows that investigating the effect of the hidden orbital polarization on antiferromagnetic information storage and processing is an important and promising theoretical and experimental future research direction.

\section{CONCLUSIONS}

In conclusion, we have shown that even in centrosymmetric, non-magnetic materials, there can exist large site-dependent, hidden orbital polarizations. In centrosymmetric group IV materials such as diamond, Si and Ge, the hidden spin polarization is very small, but the hidden orbital polarization is on the order of $\hbar$. We have also found, using a general perturbative scheme that is applicable not only to diamond, $\mathrm{Si}$ and $\mathrm{Ge}$ (with small hidden spin polarizations) but also to layered materials such as $\mathrm{MoS}_{2}$ and $\mathrm{WSe}_{2}$ with hidden spin polarizations close to the maximum value, that the hidden spin polarization is completely determined by the site-dependent orbital angular momentum in general centrosymmetric, non-magnetic materials. If the energy distance between nearby bands is comparable to or smaller than the atomic spin orbit coupling, the hidden spin polarization is large. In the case of zinc-blende materials, this energy difference (nearest-neighbor hopping) is a few $\mathrm{eV}$, and in the case of transition-metal dichalcogenides, this energy difference (interlayer hopping) is a few tens of meV. In any case, however, first-order or higher-order perturbative theory with respect to the SOC connects the hidden spin polarization to site-dependent orbital angular momenta. By comparing the strength of the SOC and the interlayer hopping constant in $\mathrm{MoS}_{2}$ and $\mathrm{WSe}_{2}$, we have shown that the hidden spin polarization in transition metal dichalcogenides can be significantly reduced by applying a pressure. Our study also illustrates that site-dependent orbital polarizations play an important role in current-induced magnetization of both centrosymmetric materials and non-centrosymmetric materials such as GaAs. We have discussed 
the experimental signatures of the hidden orbital polarization in centrosymmetric materials in both spin-resolved and -integrated photoemission spectroscopies. We have also calculated the currentdriven antiferromagnetism in compressed silicon and have shown that an appreciable amount of orbital (antiferro-)magnetization can be induced even when the spin counterpart is negligible, demonstrating the potentially important role of hidden orbital polarizations in antiferromagnetic information technology. Because there are more degrees of freedom in orbital polarization than in spin polarization, the hidden orbital polarization may lead to new discoveries in physics.

\section{CONFLICT OF INTEREST}

The authors declare no conflict of interest.

\section{ACKNOWLEDGEMENTS}

We thank Tae Yun Kim for discussions at an early stage of this work and for drawing our attention to ref 20 and Ivo Souza for discussions on many aspects of the orbital magnetization of solids and for pointing out the required symmetry lowering in the current-induced magnetization. This work was supported by the Creative-Pioneering Research Program through Seoul National University.

1 Zhang, X., Liu, Q., Luo, J.-W., Freeman, A. J. \& Zunger, A. Hidden spin polarization in inversion-symmetric bulk crystals. Nat. Phys. 10, 387 (2014).

2 Reck, R. A. \& Fry, D. L. Orbital and spin magnetization in Fe-Co, Fe-Ni, and $\mathrm{Ni}-\mathrm{Co}$. Phys. Rev. 184, 492 (1969).

3 Ceresoli, D., Gerstmann, U., Seitsonen, A. P. \& Mauri, F. First-principles theory of orbital magnetization. Phys. Rev. B 81, 060409 (2010).

4 Taylor, J. W., Duffy, J. A., Bebb, A. M., Lees, M. R., Bouchenoire, L., Brown, S. D. \& Cooper, M. J. Temperature dependence of the spin and orbital magnetization density in $\mathrm{Sm}_{0.982} \mathrm{Gd}_{0.018} \mathrm{Al}_{2}$ around the spin-orbital compensation point. Phys. Rev. B 66 161319 (2002).

5 Yoda, T., Yokoyama, T. \& Murakami, S. Current-induced orbital and spin magnetizations in crystals with helical structure. Sci. Rep. 5, 12024 (2015).

6 Zhong, S., Moore, J. E. \& Souza, I. Gyrotropic magnetic effect and the magnetic moment on the Fermi surface. Phys. Rev. Lett. 116, 077201 (2016).

7 Park, S. R., Kim, C. H., Yu, J., Han, J. H. \& Kim, C. Orbital-angular-momentum based origin of Rashba-type surface band splitting. Phys. Rev. Lett. 107, 156803 (2011).

8 Park, J.-H., Kim, C. H., Rhim, J.-W. \& Han, J. H. Orbital Rashba effect and its detection by circular dichroism angle-resolved photoemission spectroscopy. Phys. Rev. B 85, 195401 (2012)

9 Kim, B., Kim, C. H., Kim, P., Jung, W., Kim, Y., Koh, Y., Arita, M., Shimada, K., Namatame, H., Taniguchi, M., Yu, J. \& Kim, C. Spin and orbital angular momentum structure of $\mathrm{Cu}(111)$ and Au(111) surface states. Phys. Rev. B 85, 195402 (2012).

10 Baidya, S., Waghmare, U. V., Paramekanti, A. \& Saha-Dasgupta, T. High-temperature large-gap quantum anomalous Hall insulating state in ultrathin double perovskite films. Phys. Rev. B 94, 155405 (2016)

11 Vogl, P., Hjalmarson, H. P. \& Dow, J. D. A semi-empirical tight-binding theory of the electronic structure of semiconductors. J. Phys. Chem. Solids 44, 365 (1983).

12 Luttinger, J. M. Quantum theory of cyclotron resonance in semiconductors: general theory. Phys. Rev. 102, 1030 (1956)
13 Petersen, L. \& Hedegård, P. A simple tight-binding model of spin-orbit splitting of $s p$-derived surface states. Surf. Sci. 459, 49 (2000).

14 Waugh, J. A., Nummy, T., Parham, S., Liu, Q., Zhang, X., Zunger, A. \& Dessau, D. S. Minimal ingredients for orbital texture switches at Dirac points in strong spin-orbit coupled materials. arXiv:1608.01387v1 (2016).

15 Fang, S., Kuate Defo, R., Shirodkar, S. N., Lieu, S., Tritsaris, G. A. \& Kaxiras, E. $A b$ initio tight-binding Hamiltonian for transition metal dichalcogenides. Phys. Rev. B 92, 205108 (2015)

16 Zhao, Z., Zhang, H., Yuan, H., Wang, S., Lin, Y., Zeng, Q., Xu, G., Liu, Z., Solanki, G. K. Patel, K D., Cui, Y., Hwang, H Y. \& Mao, W L. Pressure induced metallization with absence of structural transition in layered molybdenum diselenide. Nat. Commun $\mathbf{6}$, 7312 (2015).

17 Chadi, D. J. Spin-orbit splitting in crystalline and compositionally disordered semiconductors. Phys. Rev. B 16, 790 (1977).

18 Murakami, S., Nagaosa, N. \& Zhang, S.-C. SU(2) non-Abelian holonomy and dissipationless spin current in semiconductors. Phys. Rev. B 69, 235206 (2004).

19 Riley, J. M., Mazzola, F., Dendzik, M., Michiardi, M., Takayama, T., Bawden, L., Granerød, C., Leandersson, M., Balasubramanian, T., Hoesch, M., Kim, T. K., Takagi, H., Meevasana, W., Hofmann, P., Bahramy, M. S., Wells, J. W. \& King, P. D. C. Direct observation of spin-polarized bulk bands in an inversionsymmetric semiconductor. Nat. Phys. 10, 835 (2014).

20 Jungwirth, T., Marti, X., Wadley, P. \& Wunderlich, J. Antiferromagnetic spintronics Nat. Nanotechnol. 11, 231 (2016).

21 Wadley, P., Howells, B., Železný, J., Andrews, C., Hills, V., Campion, R. P., Novák, V., Olejník, K., Maccherozzi, F., Dhesi, S. S., Martin, S. Y., Wagner, T., Wunderlich, J., Freimuth, F., Mokrousov, Y., Kuneš, J., Chauhan, J. S., Grzybowski, M. J., Rushforth, A. W., Edmonds, K. W., Gallagher, B. L. \& Jungwirth, T. Electrical switching of an antiferromagnet. Science 351, 587 (2016)

22 Železný, J., Gao, H., Výborný, K., Zemen, J., Mašek, J., Manchon, A., Wunderlich, J., Sinova, J. \& Jungwirth, T. Relativistic Néel-order fields induced by electrical current in antiferromagnets. Phys. Rev. Lett. 113, 157201 (2014).

23 Kugel', K. I. \& Khomskii, D. I. The Jahn-Teller effect and magnetism: transition meta compounds. Sov. Phys. Usp 25, 231 (1982).

24 Khaliullin, G. Orbital order and fluctuations in Mott insulators. Prog. Theor. Phys. Supp. 160, 155 (2005).

25 Laude, L. D., Pollak, F. H. \& Cardona, M. Effects of uniaxial stress on the indirect exciton spectrum of silicon. Phys. Rev. B 3, 2623 (1971).

26 Milne, J. S., Favorskiy, I., Rowe, A. C. H., Arscott, S. \& Renner, C. Piezoresistance in silicon at uniaxial compressive stresses up to $3 \mathrm{GPa}$. Phys. Rev. Lett. 108, 256801 (2012).

27 Harrison, W. A. Electronic Structure and the Properties of Solids, Freeman: San Francisco, USA, (1980).

$28 \mathrm{Hu}$, C. Modern Semiconductor Devices for Integrated Circuits, Prentice Hall: New Jersey, USA, (2010)

29 Wiegelmann, H., Jansen, A. G. M., Wyder, P., Rivera, J. P. \& Schmid, H. Magnetoelectric effect of $\mathrm{Cr}_{2} \mathrm{O}_{3}$ in strong static magnetic fields. Ferroelectrics 162, 141 (1994).

(i) This work is licensed under a Creative Commons Attribution 4.0 International License. The images or other third party material in this article are included in the article's Creative Commons license, unless indicated otherwise in the credit line; if the material is not included under the Creative Commons license, users will need to obtain permission from the license holder to reproduce the material. To view a copy of this license, visit http:// creativecommons.org/licenses/by/4.0/

(C) The Author(s) 2017

Supplementary Information accompanies the paper on the NPG Asia Materials website (http://www.nature.com/am) 